\title{
Quality of life in patients with diabetic nephropathy: findings from the KNOW-CKD (Korean Cohort Study for Outcomes in Patients with Chronic Kidney Disease) cohort
}

\author{
Hyunsuk Kim ${ }^{1}$, Joongyub Lee ${ }^{2}$, Gwang Ho Choi $^{1}$, Hae Min Jeong ${ }^{1}$, Seok hyung Kim ${ }^{1}$, Jae Eon Gu$^{1}$, Jeong-Ju Yoo ${ }^{3}$, \\ Miyeun $\mathrm{Han}^{4}$, Hyo-Jin $\mathrm{Kim}^{4}$, Su-Ah Sung ${ }^{5}$, Seung Hyeok Han ${ }^{6}$, Yeong Hoon Kim ${ }^{7}$, Jong-Woo Yoon ${ }^{1}$, Jongho Heo , \\ Kook-Hwan $\mathrm{Oh}^{9}$ \\ ${ }^{1}$ Department of Internal Medicine, Hallym University Chuncheon Sacred Heart Hospital, Chuncheon, Republic of Korea \\ ${ }^{2}$ Prevention and Management Center, Inha University Hospital, Incheon, Republic of Korea \\ ${ }^{3}$ Department of Internal Medicine, Soonchunhyang University Hospital Bucheon, Bucheon, Republic of Korea \\ ${ }^{4}$ Department of Internal Medicine, Pusan National University Hospital, Busan, Republic of Korea \\ ${ }^{5}$ Department of Internal Medicine, Eulji General Hospital, Seoul, Republic of Korea \\ ${ }^{6}$ Department of Internal Medicine, Institute of Kidney Disease Research, Yonsei University College of Medicine, Seoul, Republic of Korea \\ ${ }^{7}$ Department of Internal Medicine, Inje University Busan Paik Hospital, Inje University College of Medicine, Busan, Republic of Korea \\ ${ }^{8}$ National Assembly Futures Institute, Seoul, Republic of Korea \\ ${ }^{9}$ Department of Internal Medicine, Seoul National University Hospital, Seoul, Republic of Korea
}

Background: Diabetic nephropathy (DN) can affect quality of life (QoL) because it requires arduous lifelong management. This study analyzed QoL differences between DN patients and patients with other chronic kidney diseases (CKDs).

Methods: The analysis included subjects $(n=1,766)$ from the KNOW-CKD (Korean Cohort Study for Outcomes in Patients with Chronic Kidney Disease) cohort who completed the Kidney Disease Quality of Life Short Form questionnaire. After implementing propensity score matching (PSM) using factors that affect the QoL of DN patients, QoL differences between DN and non-DN participants were examined. Results: Among all DN patients $(n=390)$, higher QoL scores were found for taller subjects, and lower scores were found for those who were unemployed or unmarried, received Medical Aid, had lower economic status, had higher platelet counts or alkaline phosphatase levels, or used clopidogrel or insulin. After PSM, the 239 matched DN subjects reported significantly lower patient satisfaction (59.9 vs. $64.5, p=0.02$ ) and general health (35.3 vs. 39.1, $p=0.04)$ than the 239 non-DN subjects. Scores decreased in both groups during the 5-year follow-up, and the scores in the work status, sexual function, and role-physical domains were lower among DN patients than non-DN patients, though those differences were not statistically significant.

Conclusion: Socioeconomic factors of DN were strong risk factors for impaired QoL, as were high platelet, alkaline phosphatase, and clopidogrel and insulin use. Clinicians should keep in mind that the QoL of DN patients might decrease in some domains compared with non-DN CKDs.

Keywords: Diabetic nephropathy, Kidney disease component summary, Kidney Disease Quality of Life Short Form questionnaire, Mental component summary, Physical component summary, Quality of life

Received: March 23, 2021; Revised: June 13, 2021; Accepted: June 27, 2021

Correspondence: Kook-Hwan Oh

Department of Internal Medicine, Seoul National University College of Medicine, 101 Daehak-ro, Jongno-gu, Seoul 03080, Republic of Korea. E-mail: ohchris@hanmail.net

ORCID: https://orcid.org/0000-0001-9525-2179

Copyright (C) 2022 by The Korean Society of Nephrology

(a) This is an Open Access article distributed under the terms of the Creative Commons Attribution Non-Commercial and No Derivatives License (http:// creativecommons.org/licenses/by-nc-nd/4.0/) which permits unrestricted non-commercial use, distribution of the material without any modifications, and reproduction in any medium, provided the original works properly cited. 


\section{Introduction}

Diabetic nephropathy (DN) is the top cause of end-stage renal disease (ESRD). In South Korea, $48.8 \%$ of ESRD is caused by DN (http://www.ksn.or.kr/rang_board/list.html? code=sinchart; current renal replacement therapy in Korea, Insan Memorial Dialysis Registry 2019 [1]), and DN is also a major component of the global burden of ESRD.

DN can affect quality of life (QoL) because it requires arduous lifelong management. Various studies have examined factors that correlate with low QoL in DN patients. Doctors cannot consider patients' short-term QoL; instead, they must focus on reducing patients' risk of complications to improve their long-term QoL [2]. For example, glucose fluctuations affect cognitive function among patients with diabetes mellitus, which can lead to diminished QoL [3]. Among patients with diabetes, QoL is lower in women than in men, especially in terms of mental health. QoL was found to be worse among young women than among men with high hemoglobin Alc (HbAlc) levels [4]. Another study reported that QoL was better when patients were younger, their diabetes diagnosis had been made in the past 5 years, their HbAlc level was lower than $7 \%$, they had no other complications or history of hospitalization, and their body mass index (BMI) was below $24 \mathrm{~kg} / \mathrm{m}^{2}$ [5].

However, how the QoL of patients with DN compares with that of patients with other types of chronic kidney disease (CKD) is not well known. Therefore, we used data from the KNOW-CKD (Korean Cohort Study for Outcomes in Patients with Chronic Kidney Disease) cohort, which includes multiple types of CKD (glomerulonephritis, DN, hypertensive kidney disease, polycystic kidney disease, etc.), to investigate factors that influence the QoL of patients with DN and determine whether the QoL of patients with DN differs from that of other patients in the KNOW-CKD cohort. To accomplish that goal, we compared QoL changes from baseline to 5 years later between $\mathrm{DN}$ and non-DN subjects.

\section{Methods}

\section{Study population and ethics statement}

This study used data from KNOW-CKD, a multicenter prospective cohort study that included patients from through- out Korea who were 20 to 75 years old with stage 1 to 5 CKD and not on dialysis. The design and methods of the KNOW-CKD study have been presented in detail elsewhere (NCT01630486 at http://www.clinicaltrials.gov) [6]. All procedures were conducted in accordance with the relevant ethical standards of the institutional and national research committees and with the 1964 Helsinki Declaration and its later amendments or comparable ethical standards. Informed consent was obtained from all subjects. The Institutional Review Board of each clinical center from which patients were drawn approved the KNOW-CKD study protocol in 2013: Seoul National University Hospital (No. 1104089-359), Seoul National University Bundang Hospital (No. B-1106/129-008), Yonsei University Severance Hospital (No. 4-2011-0163), Kangbuk Samsung Medical Center (No. 201101-076), The Catholic University of Korea, Seoul St. Mary's Hospital (No. KC11OIMI0441), Gachon University Gil Hospital (No. GIRBA2553), Eulji General Hospital (No. 201105-01), Chonnam National University Hospital (No. CNUH-2011092), and Inje University Busan Paik Hospital (No. 11-091).

\section{Data collection and variables}

For the present study, data were searched using an electronic data management system (PhactaX, Seoul, Republic of Korea) with assistance from the data management department of the Medical Research Collaborative Center at Seoul National University. The following variables were analyzed: sex; age; weight; height; BMI; blood pressure; number of family members; work, marital, insurance, and economic status; coronary artery calcium score; hemoglobin; platelet count; albumin, uric acid, calcium, blood urea nitrogen (BUN), phosphate, alkaline phosphatase, total bilirubin, fasting glucose, HbAlc, vitamin D3, creatinine (Cr), and cystatin C (Cys C) levels; iron and total iron binding capacity (TIBC); hepcidin, adiponectin, and klotho levels; relative wall thickness (RWT); and use of clopidogrel or insulin. Serum Cr was measured using traceable isotope-dilution mass spectrometry. Samples collected before May 1, 2013, were analyzed retrospectively on the basis of samples stored in the Biobank. After May 1, 2013, real-time measurements were made after samples were collected from each institution and transported to a central laboratory. 


\section{Definitions}

The Korean version 1.3 of the Kidney Disease Quality of Life Short Form (KDQOL-SF) was used to evaluate health-related QoL (HRQoL). The KDQOL-SF contains disease-targeted items (kidney disease component summary, KDCS) focused on the particular health-related concerns of subjects with kidney disease who are on dialysis: (1) symptoms/problems, (2) effects of kidney disease on daily life, (3) burden of kidney disease, (4) work status, (5) cognitive function, (6) quality of social interactions, (7) sexual function, (8) sleep, (9) social support, (10) dialysis staff encouragement, and (11) patient satisfaction. The KDQOL-SF also contains the Medical Outcome Study Short Form-36 Health Survey (SF36), which is composed of a physical component summary (PCS) and a mental component summary (MCS). The PCS and MCS each contain four subscales: (1) physical function, (2) role-physical (limitations due to physical problems), (3) bodily pain, and (4) general health and (1) vitality, (2) role-emotional, (3) social function, and (4) mental health, respectively. Within the KDQOL-SF, each domain of the KDCS and SF-36 is scored on a 100-point scale, with higher scores representing better QoL. The scores of items in the kidney disease-specific part of the KDQOL-SF were averaged to give the KDCS score. The scores from the SF-36 part of the KDQOL-SF were summarized as MCS and PCS. The number of family members was grouped into $\leq 1,2-3$, or $>3$. Work status was defined as employed or unemployed; marital status was divided into married, unmarried, and divorced or widowed; economic status was divided into higher (>\$4,000/family/month), middle ( $\$ 1,330-\$ 4,000 /$ family/ month) and lower ( $<\$ 1,330 /$ family/month); and insurance status was divided into medical insurance or Medical Aid (including refunds). The coronary artery calcium score was divided into two groups ( $\leq 400$ and $>400$ ). RWT allows further classification of left ventricular mass increase as either concentric hypertrophy (RWT $>0.42$ ) or eccentric hypertrophy (RWT $\leq 0.42$ ). DN was defined as CKD primarily or exclusively caused by diabetes. Among the 2,238 subjects in the KNOW-CKD cohort, 754 patients (33\%) had diabetes, and 519 (23\%) were deemed to have DN. In the KNOW-CKD cohort, the most common disease was glomerulonephritis (36\%), followed by DN (23\%), hypertensive nephropathy (18\%), autosomal dominant polycystic kidney disease (16\%), and other unclassified CKD (6\%). In this study, the non-DN group contained all DN patients in the KNOW-CKD cohort not diagnosed with diabetes.

\section{The process of propensity score matching}

This study analyzed the 1,766 KNOW-CKD subjects who completed the KDQOL-SF. First, factors that influenced the KDCS, PCS, and MCS scores among the subjects with DN (n $=390$ ) were examined. Then, we conducted propensity score matching (PSM) to control the factors that were significant in the DN subjects, reduce the effects of selection bias, and potential confounding between the two groups $[7,8]$. The following 32 variables were used for PSM: sex; age; weight; height; BMI; blood pressure, smoking; number of family members; work, marital, insurance, and economic status; coronary artery calcium score; RWT; Cr; Cys C; hemoglobin; platelet count; albumin, uric acid, calcium, BUN, phosphate, alkaline phosphatase, total bilirubin, and vitamin D3 levels; iron; TIBC; hepcidin, adiponectin, and klotho levels; and use of clopidogrel. To measure the balancing, we calculated the standardized bias for each measured covariate in the weighted samples using the "twang" package in R version 4.0.4 (R Foundation for Statistical Computing, Vienna, Austria; http://cran.r-project.org/). After PSM, 239 DN and 239 nonDN subjects were successfully selected (Supplementary Fig. 1 , available online). Differences between the two groups in mean baseline and 5-year follow-up KDQOL-SF scores were then analyzed (Fig. 1).

\section{Statistical methodology}

Variables are expressed as the mean \pm standard deviation or number (\%). The p-value were calculated by Student's t-test or Mann-Whitney U test for continuous variables and Chisquare test or Fisher's exact test for categorical variables. First, meaningful factors were selected from the KNOWCKD dataset using a literature review of factors that affect QoL in DN. Then, a linear regression model was constructed with those factors to understand which ones influenced QoL in the subjects with DN in the KNOW-CKD cohort. Factors that were significant $(\mathrm{p}<0.05)$ in the univariate analysis were used in the multivariate model, and KDCS, PCS, and MCS were analyzed separately. After selecting the DN and non-DN groups through PSM by considering the variables found to influence the QoL of DN patients, the differences 


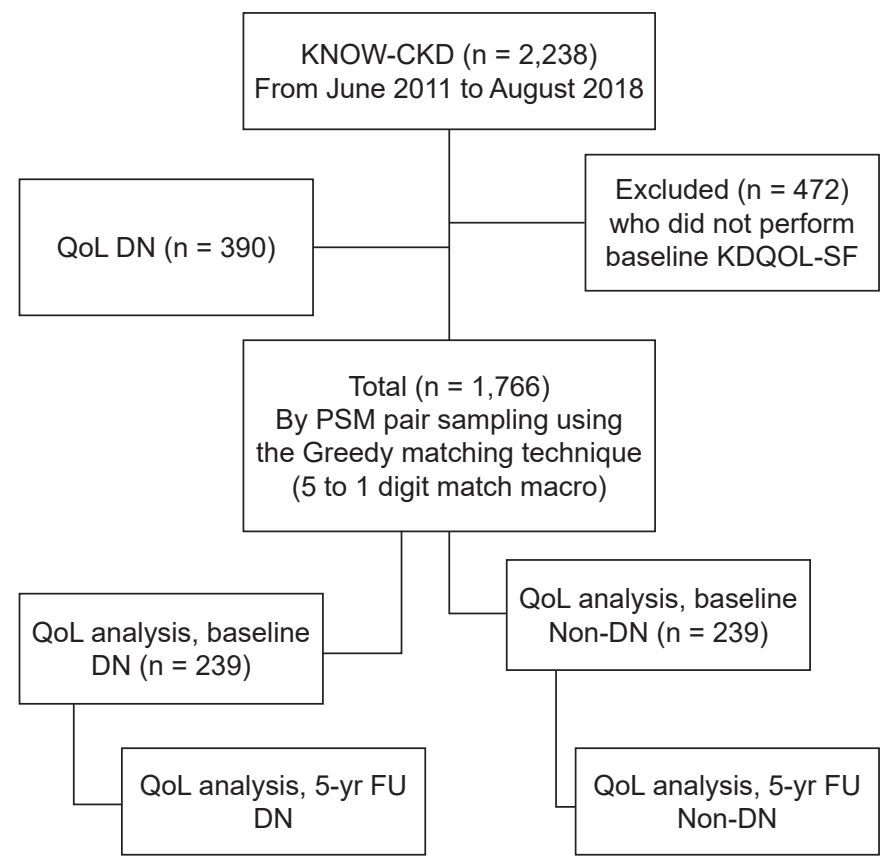

Figure 1. Flow chart of the patients. The analysis incorporated the 1,766 KNOW-CKD (Korean Cohort Study for Outcomes in Patients with Chronic Kidney Disease) subjects who completed the Kidney Disease Quality of Life Short Form (KDQOL-SF). After finding the factors that significantly influenced the kidney disease component summary, physical component summary, and mental component summary scores of subjects with diabetic nephropathy (DN) $(n=390)$, propensity score matching (PSM) using those factors yielded $239 \mathrm{DN}$ and 239 non-DN subjects. Between-group differences in mean baseline and 5-year follow-up (FU) KDQOL-SF scores were analyzed.

QoL, quality of life.

between the two groups in their baseline and 5-year follow-up KDQOL-SF scores were analyzed using the Student $t$ test. Changes in the KDQOL-SF scores over 5 years were also compared between the two groups using the Student $t$ test. All statistical analyses were conducted using IBM SPSS version 23.0 for Windows (IBM Corp., Armonk, NY, USA).

\section{Results}

\section{Baseline characteristics of diabetic nephropathy subjects}

Data from 390 DN patients were used to analyze QoL. Among them, 276 (70.8\%) were male, and their average age was 59 years. Their average height was $165 \mathrm{~cm}$ and average BMI was $25.4 \mathrm{~kg} / \mathrm{m}^{2}$. One hundred forty-two (36.4\%) had more than three family members, and $46.4 \%(\mathrm{n}=181)$ of them were working. Furthermore, 320 (82.3\%) were married, and more than $50 \%$ of them were in the middle economic status group. Approximately one-third $(32.5 \%, \mathrm{n}=119)$ of the participants had a coronary calcium score greater than 400. The average $\mathrm{Cr}$ level was $2.4 \mathrm{mg} / \mathrm{dL}$, albumin level was $4.0 \mathrm{~g} / \mathrm{dL}$, and hemoglobin level was $11.7 \mathrm{~g} / \mathrm{dL}$. The average platelet count was 241,000 per microliter, and HbAlc was 7.3\%. Thirty-five of the DN patients (9.0\%) used clopidogrel, and 223 (57.3\%) used insulin (Table 1). Before PSM, most of those variables differed between the DN and non-DN subjects (Table 1).

Linear regression analysis of the quality of life of diabetic nephropathy subjects

To understand which factors influenced the QoL of subjects with $\mathrm{DN}$, linear regression analyses were conducted using the KDCS, PCS, and MCS scores and other factors. The univariate analysis of the KDCS scores found lower scores in women and higher scores in those with greater weight or height, although there was no association with BMI. Regarding socioeconomic factors, scores were higher in those with more than three family members and lower in those who were unemployed; unmarried, divorced, or widowed; without Medical Aid, and in the lower economic status group. Lower KDCS scores were also associated with higher levels of $\mathrm{Cr}$, Cys C, platelets, BUN, phosphate, alkaline phosphatase, fasting glucose, HbAlc, and hepcidin; lower levels of hemoglobin, calcium, total bilirubin, vitamin D3, iron, and TIBC; and use of clopidogrel or insulin. In the multivariate model, KDCS scores were lower in subjects who had shorter height (B [95\% confidence interval], 0.34 [0.09-0.60]), were unemployed (-6.02 [-8.70 to -3.34$])$, had Medical Aid (-10.29 [-16.51 to $-4.06])$, and had higher levels of alkaline phosphatase $(-0.02$ [-0.04 to -0.004$])$. The scores were also lower among subjects who used clopidogrel (no use, 4.86 [0.47-9.25]) (Supplementary Table 1, available online; Fig. 2A).

In the univariate analysis of PCS scores, lower scores were found in women, and higher scores were found in those with greater height. Regarding socioeconomic factors, low QoL was found among the unemployed, unmarried, those without Medical Aid, and those with a lower economic status. No association was observed for coronary artery calcium score, but the score was lower for participants with an RWT $>0.42$. 
Table 1. Baseline characteristics of diabetic nephropathy and non-diabetic nephropathy subjects before propensity score matching

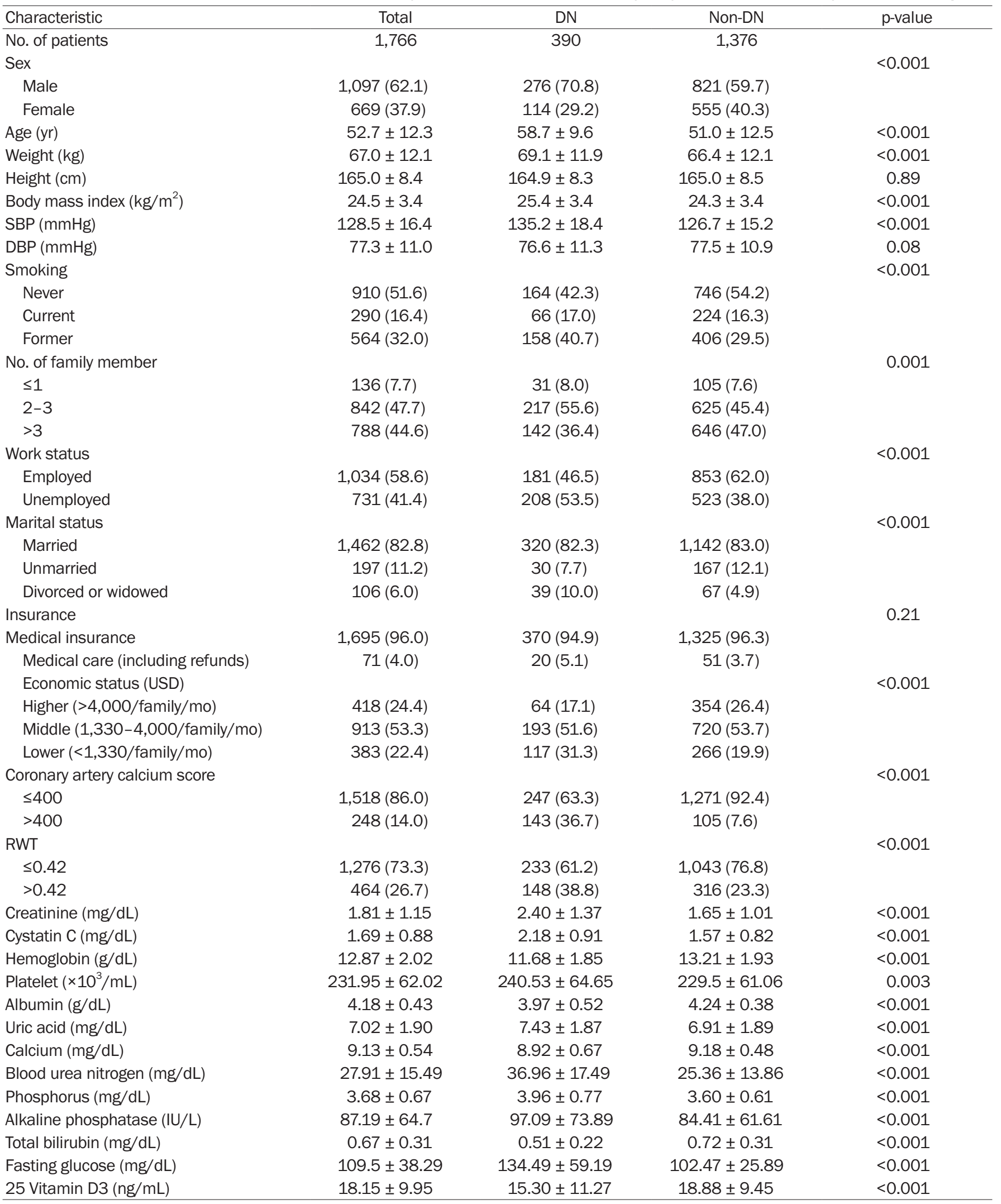


Table 1. Continued

\begin{tabular}{|c|c|c|c|c|}
\hline Characteristic & Total & DN & Non-DN & $\mathrm{p}$-value \\
\hline Iron $(\mu \mathrm{g} / \mathrm{dL})$ & $92.66 \pm 35.59$ & $81.30 \pm 28.38$ & $95.89 \pm 36.76$ & $<0.001$ \\
\hline $\mathrm{TIBC}(\mu \mathrm{g} / \mathrm{dL})$ & $297.30 \pm 53.01$ & $280.25 \pm 51.04$ & $302.14 \pm 52.58$ & $<0.001$ \\
\hline Hepcidin (ng/mL) & $18.39 \pm 18.41$ & $24.12 \pm 21.12$ & $16.76 \pm 17.23$ & $<0.001$ \\
\hline Adiponectin $(\mu \mathrm{g} / \mathrm{mL})$ & $11.79 \pm 9.91$ & $13.22 \pm 11.4$ & $11.39 \pm 9.41$ & 0.02 \\
\hline Klotho (pg/mL) & $547.06 \pm 326.17$ & $530.16 \pm 213.29$ & $551.85 \pm 351.55$ & 0.41 \\
\hline Urine protein/creatinine $(\mathrm{g} / \mathrm{g})$ & $1.35 \pm 1.9$ & $2.84 \pm 2.86$ & $0.95 \pm 1.3$ & $<0.001$ \\
\hline Clopidogrel & & & & $<0.001$ \\
\hline Yes & $76(4.3)$ & $35(9.0)$ & $41(3.0)$ & \\
\hline No & $1,689(95.7)$ & $354(91.0)$ & 1,335 (97.0) & \\
\hline Insulin & & & & $<0.001$ \\
\hline Yes & $223(12.6)$ & $223(57.3)$ & $0(0)$ & \\
\hline No & $1,543(87.4)$ & $166(42.7)$ & $0(0)$ & \\
\hline
\end{tabular}

Data are expressed as number only, number (\%), or mean \pm standard deviation.

DBP, diastolic blood pressure; DN, diabetic nephropathy; RWT, relative wall thickness; SBP, systolic blood pressure; TIBC, total iron binding capacity; USD, U.S. dollar.

Lower scores were associated with higher levels of Cr, Cys C, platelets, BUN, phosphate, $\mathrm{HbAlc}$, adiponectin, and urinary protein/Cr ratio and with lower levels of hemoglobin, albumin, calcium, total bilirubin, and iron. Lower scores were also found among participants using clopidogrel or insulin. In the multivariate model, PCS was lower among participants in the lower economic status group $(-5.87[-12.43$ to $0.68])$ and in those with high platelet counts $(-0.05[-0.08$ to $-0.01])$ and alkaline phosphatase $(-0.03$ [-0.07 to -0.002$])$ levels. Low PCS was also associated with clopidogrel use (no use, 9.383 [2.127-16.639]) (Supplementary Table 2, available online; Fig. 2B).

In the univariate analysis of MCS scores, lower scores were found among women and those with a higher BMI, and higher scores were associated with greater height. Regarding socioeconomic factors, scores were higher among former smokers, and QoL was lower among patients in the middle and lower economic status groups than among those who were unemployed, did not have Medical Aid, or were in the higher economic status group. The coronary artery calcium score did not show a significant association, but low scores were associated with an RWT $>0.42$. Lower scores were found in patients with higher levels of $\mathrm{Cr}$, platelets, phosphate, alkaline phosphatase, fasting glucose, HbAlc, adiponectin, and urinary protein/Cr ratio and in patients with lower levels of hemoglobin, total bilirubin, iron, and TIBC. Scores were also lower among participants who used clopidogrel or insulin. In the multivariate model, lower MCS scores were found among participants with lower economic status ( -7.58 [-14.34 to -0.82$])$ and those with a high platelet count $(-0.05$ [-0.08 to -0.01$])$ (Supplementary Table 3, available online; Fig. 2C).

In summary, QoL among patients with $\mathrm{DN}$ was higher in taller participants and lower in subjects who were unemployed, unmarried, without Medical Aid, or in the lower economic status group, as shown consistently in the KDCS, PCS, and MCS scores. Interestingly, lower QoL was also found in patients with higher alkaline phosphatase levels and platelet counts. As expected, QoL was low among participants using clopidogrel or insulin.

\section{Baseline characteristics of subjects in the diabetic nephropathy and non-diabetic nephropathy groups after propensity score matching}

PSM was conducted to minimize the differences between the DN and non-DN groups. The variables used for PSM were selected from the factors found to influence DN in the univariate analyses. Ultimately, 239 participants were allocated to the DN and non-DN groups, respectively. Due to the clinical characteristics of $\mathrm{DN}$, fasting glucose and the spot urine protein/Cr ratio were not included as PSM variables. Because the two groups were balanced through PSM, none of the included variables differed significantly between them at baseline (Table 2).

Approximately two-thirds (66\%) of the patients were male, with an average age of around 58 years. Their average height was $164 \mathrm{~cm}$, their average BMI was roughly $25 \mathrm{~kg} / \mathrm{m}^{2}$, and 

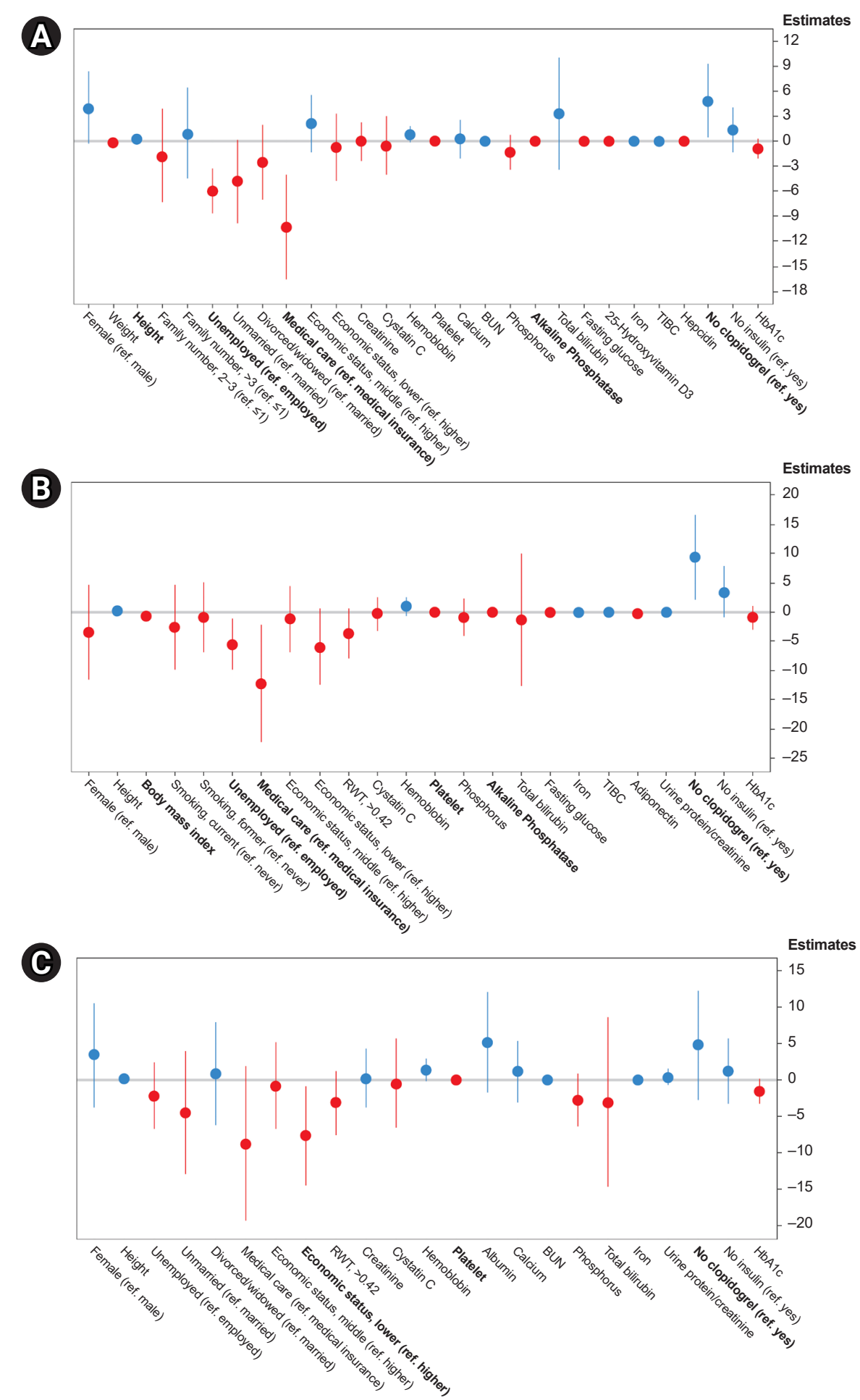

Figure 2. Forest plot of linear regression for KDCS, PCS, and MCS scores in the DN group. (A) Linear regression analyses of KDCS, PCS, and MCS scores and QoL-influencing factors were conducted. Lower KDCS scores were associated with short stature, unemployment, unmarried status, non-Medical Aid status, higher alkaline phosphatase levels, and clopidogrel use. (B) Lower PCS scores were associated with lower economic status, high platelet counts, high alkaline phosphatase levels, and clopidogrel use. (C) Lower MCS scores were associated with lower economic status and high platelet counts.

BUN, blood urea nitrogen; DN, diabetic nephropathy; HbA1c, hemoglobin A1c; KDCS, kidney disease component summary; MCS, mental component summary; PCS, physical component summary; QoL, quality of life; ref., reference; RWT, relative wall thickness; TIBC, total iron binding capacity. 
their average systolic and diastolic blood pressure was 132 $\mathrm{mmHg}$ and $77 \mathrm{mmHg}$, respectively.

More than half (53.2\%) of the participants had a history of smoking, $63.0 \%$ had more than three family members, $47.9 \%$ were employed, and $82.0 \%$ were married. Furthermore, 93.5\% had medical insurance, and $6.5 \%$ were covered under Medical Aid. The middle economic status $(\$ 1,330-\$ 4,000$ / family/month) included $49.0 \%$ of the participants. A coronary artery calcium score $>400$ was present in $22 \%$ of the participants, and their mean $\mathrm{Cr}$, hemoglobin, platelet count, albumin, uric acid, and calcium levels were $2.3 \mathrm{mg} / \mathrm{dL}, 12.0$ $\mathrm{g} / \mathrm{dL}, 232,000 / \mu \mathrm{L}, 4.1 \mathrm{~g} / \mathrm{dL}, 7.4 \mathrm{mg} / \mathrm{dL}$, and $9.0 \mathrm{mg} / \mathrm{dL}$, respectively. Around 5\% used clopidogrel (Table 2).

Table 2. Baseline characteristics of diabetic nephropathy and non-diabetic nephropathy subjects after propensity score matching

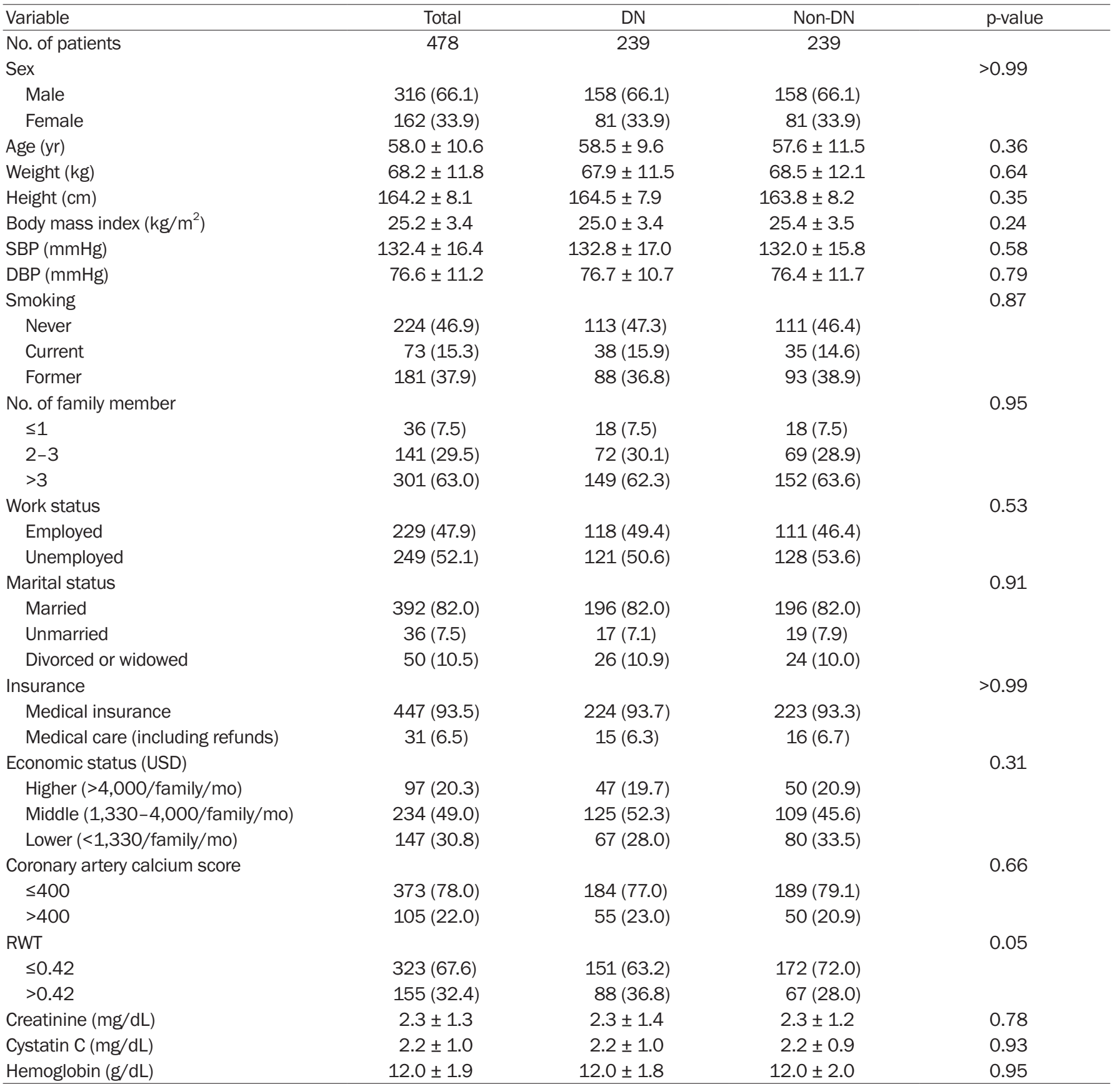


Table 2. Continued

\begin{tabular}{|c|c|c|c|c|}
\hline Variable & Total & DN & Non-DN & p-value \\
\hline Platelet $\left(\times 10^{3} / \mathrm{mL}\right)$ & $231.5 \pm 65.3$ & $231.9 \pm 64.5$ & $230.1 \pm 65.4$ & 0.14 \\
\hline Albumin (g/dL) & $4.1 \pm 0.5$ & $4.1 \pm 0.5$ & $4.1 \pm 0.4$ & 0.55 \\
\hline Uric acid (mg/dL) & $7.4 \pm 1.8$ & $7.3 \pm 1.8$ & $7.5 \pm 1.8$ & 0.19 \\
\hline Calcium (mg/dL) & $9.0 \pm 0.6$ & $9.0 \pm 0.7$ & $9.0 \pm 0.6$ & 0.96 \\
\hline Blood urea nitrogen $(\mathrm{mg} / \mathrm{dL})$ & $35.3 \pm 17.1$ & $35.3 \pm 17.0$ & $35.2 \pm 17.2$ & 0.92 \\
\hline Phosphorus (mg/dL) & $3.9 \pm 0.7$ & $3.9 \pm 0.7$ & $3.9 \pm 0.7$ & 0.49 \\
\hline Alkaline phosphatase (IU/L) & $98.1 \pm 70.7$ & $101.7 \pm 74.4$ & $94.4 \pm 66.7$ & 0.26 \\
\hline Total bilirubin (mg/dL) & $0.6 \pm 0.3$ & $0.6 \pm 0.3$ & $0.6 \pm 0.3$ & 0.05 \\
\hline Fasting glucose (mg/dL) & $123.2 \pm 52.9$ & $130.3 \pm 55.8$ & $116.0 \pm 48.8$ & $0.003 *$ \\
\hline 25 Vitamin D3 (ng/mL) & $17.0 \pm 7.3$ & $16.8 \pm 7.4$ & $17.3 \pm 7.2$ & 0.41 \\
\hline Iron ( $\mu \mathrm{g} / \mathrm{dL})$ & $86.7 \pm 32.1$ & $84.9 \pm 30.9$ & $88.6 \pm 33.3$ & 0.22 \\
\hline TIBC $(\mu \mathrm{g} / \mathrm{dL})$ & $287.3 \pm 53.8$ & $283.0 \pm 49.2$ & $291.7 \pm 57.8$ & 0.08 \\
\hline Hepcidin (ng/mL) & $22.7 \pm 21.2$ & $23.3 \pm 20.8$ & $22.2 \pm 21.6$ & 0.59 \\
\hline Adiponectin, $(\mu \mathrm{g} / \mathrm{mL})$ & $12.8 \pm 10.8$ & $12.3 \pm 11.0$ & $13.2 \pm 10.6$ & 0.38 \\
\hline Klotho (pg/mL) & $520.3 \pm 234.8$ & $515.1 \pm 208.5$ & $525.5 \pm 258.8$ & 0.63 \\
\hline Urine protein/creatinine (g/g) & $1.9 \pm 2.5$ & $2.3 \pm 2.7$ & $1.5 \pm 2.2$ & $>0.99 *$ \\
\hline Clopidogrel & & & & 0.31 \\
\hline Yes & $26(5.4)$ & $16(6.7)$ & $10(4.2)$ & \\
\hline No & $452(94.6)$ & $223(93.3)$ & $229(95.8)$ & \\
\hline
\end{tabular}

Data are expressed as number only, number (\%), or mean \pm standard deviation.

DBP, diastolic blood pressure; DN, diabetic nephropathy; RWT, relative wall thickness; SBP, systolic blood pressure; TIBC, total iron binding capacity; USD, U.S. dollar.

$* p<0.05$.

\section{Baseline quality of life scores in the two groups}

For the entire matched sample of 478 subjects, each domain of the KDQOL-SF scores was analyzed and visualized in a radar chart. For the domains of symptoms/problem list, physician encouragement, effects of kidney disease, cognitive function, physical function, and social function, the average scores were greater than 80 . However, the average scores for work status, sexual function, patient satisfaction, and burden of kidney disease were less than 65 . When the two groups were compared, the patient satisfaction score (DN vs. nonDN: 59.9 vs. $64.5, p=0.022$ ) was significantly lower in the DN group than in the non-DN group. Additionally, the sexual function (31.8 vs. 37.0 ) and work status scores (50.7 vs. 55.0) tended to be lower in the DN group, but those differences were not statistically significant.

When the SF-36 domains were analyzed, the scores for physical function, pain, and social function were greater than 80 , but the scores for general health, emotional well-being, and energy/function were lower than 65 . The general health score (35.3 vs. 39.1, $\mathrm{p}=0.04$ ) in the DN group was significantly lower than that in the non-DN group. Additionally, the energy/fatigue score (46.2 vs. 49.6 ) tended to be lower in the DN group than in the non-DN group, but that difference was not statistically significant. Regardless of statistical significance, the absolute scores in most domains was lower in the DN group than in the non-DN group. The DN group also tended to have lower KDCS, PCS, and MCS scores, although that tendency was not statistically significant between the two groups (Supplementary Table 4, available online; Fig. 3).

\section{Comparison of 5-year follow-up quality of life scores between the two groups}

In the total sample of 478 matched subjects, the QoL scores of 221 participants ( $\mathrm{DN}=100$, non-DN $=121$ ) who completed an additional 5-year follow-up QoL questionnaire were analyzed. The scores for work status and sexual function were lower in the DN group than in the non-DN group, but that difference was not statistically significant. In the SF36 , the DN group had lower role-physical domain scores, but that was not statistically significant either. The absolute composite scores in each group tended to be lower in the DN group, but the difference was not statistically significant (Supplementary Table 4, Fig. 4). 
A

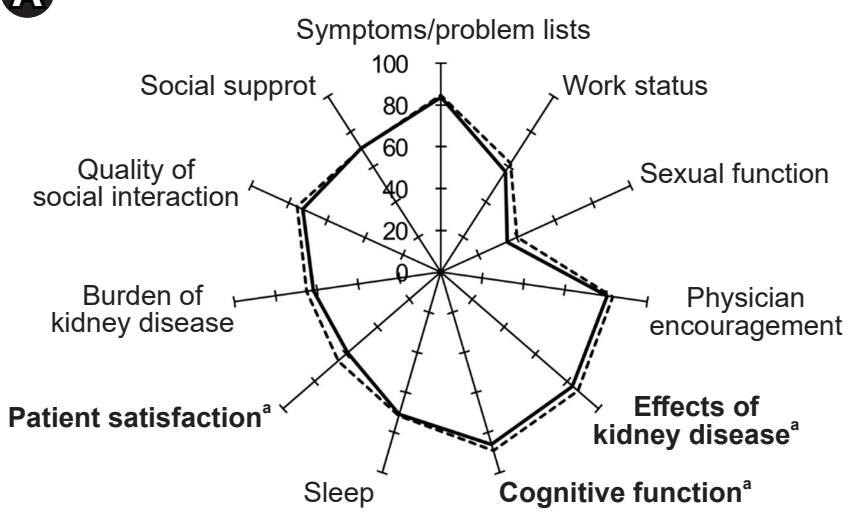

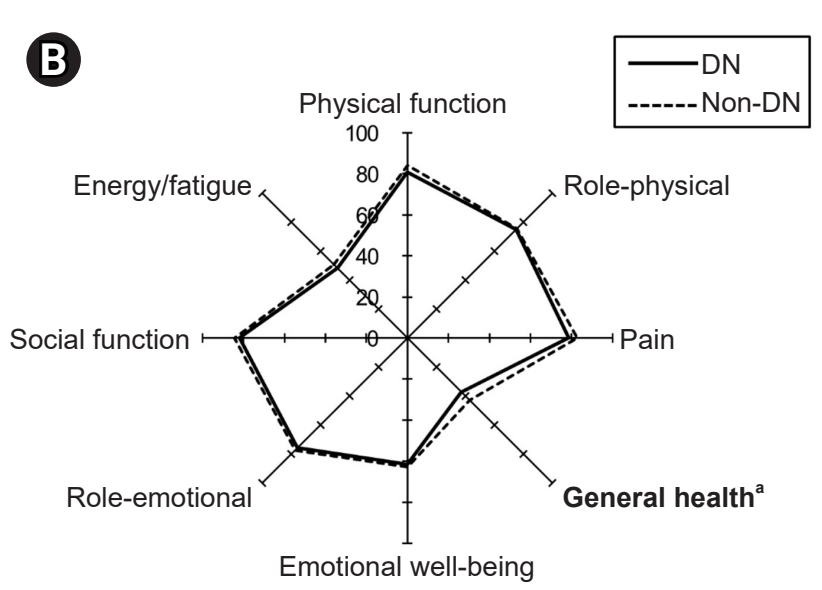

Emotional well-being

C

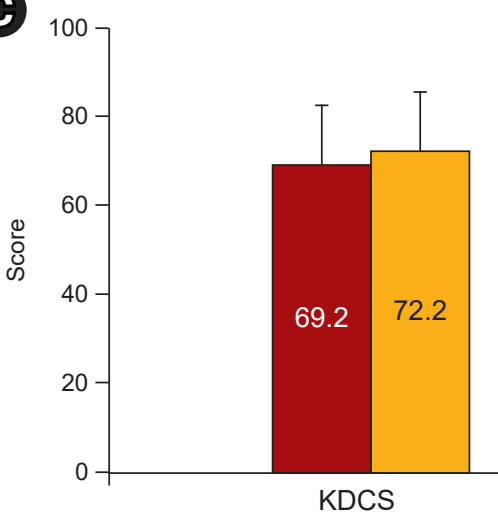
. 
(A)

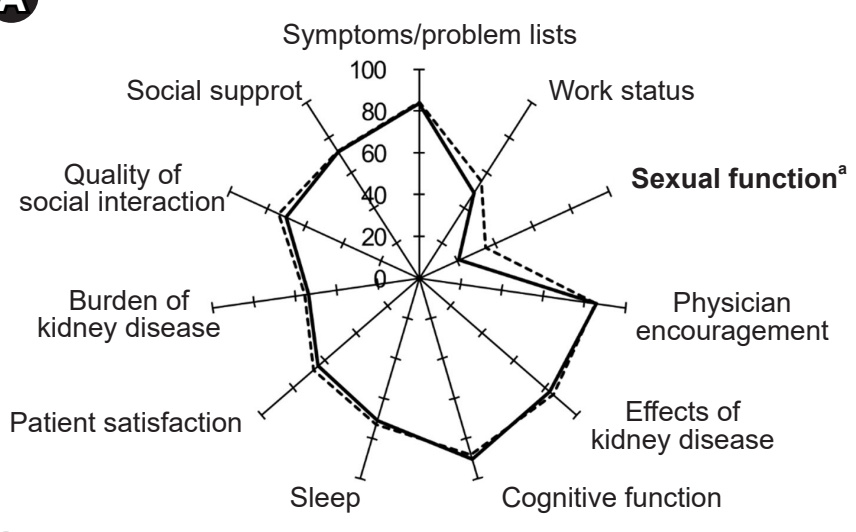

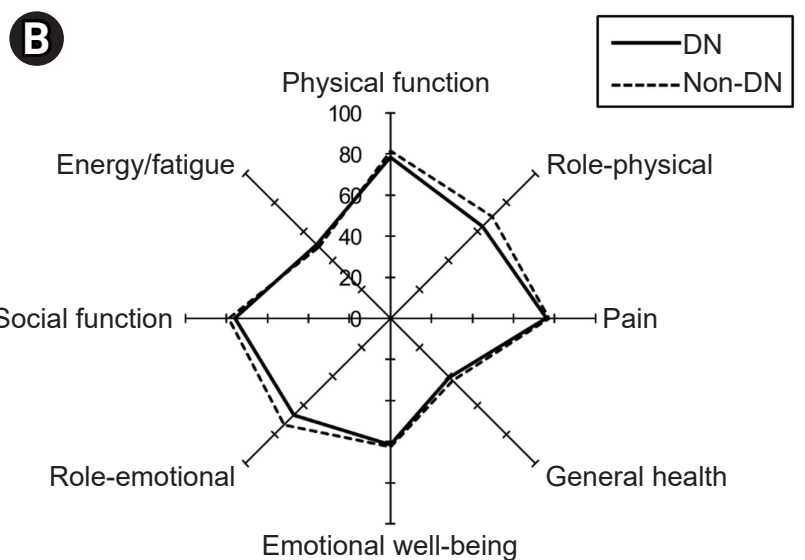

Emotional well-being

C

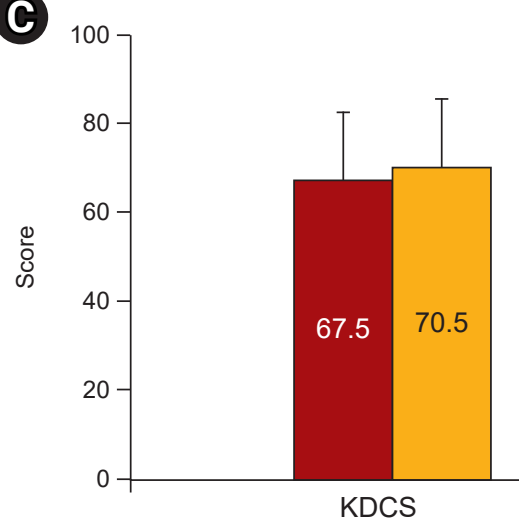

Figure 4. Spider chart of follow-up data. (A) Differences of each component of kidney disease component summary (KDCS) according to the two groups. (B) Differences of each component of mental component summary (MCS) and physical component summary (PCS) according to the two groups. (C) Summarizing KDCS, MCS, and PCS according to the two groups. The diabetic nephropathy (DN) group had non-significantly lower work status, sexual function, role-physical domain, and composite scores.

${ }^{a}$ Significant between-group difference.

tion, and role-emotional domains was greater among DN patients than among non-DN patients, but the difference was not statistically significant. In the composite scores, each group saw a decline in QoL during the 5-year follow-up period, and the difference between the two groups was not statistically significant (Supplementary Table 4, Fig. 5).

\section{Discussion}

We began this study by wondering whether DN impairs QoL more than other types of CKD because a preliminary analysis showed that patients with DN had a significantly lower baseline HRQoL than other patients in the KNOW-CKD cohort. The KNOW-CKD cohort includes patients with various types of CKD [6], but it was difficult to match group characteristics because the DN group had more advanced-stage and elderly patients than the general cohort. Therefore, we analyzed QoL among DN patients after controlling for physical and social factors that influenced QoL. When we compared the DN and non-DN groups selected through PSM, we confirmed that the DN group had inferior QoL in certain domains. In the baseline QoL, for which the sample size was sufficient, the DN group had lower scores than the non-DN group in the effects of kidney disease, cognitive function, and general health. In the 5-year follow-up data, the DN group had lower scores for sexual function than the non-DN group. In both groups, QoL decreased during the 5-year follow-up. In a few domains, the QoL score was lower among non-DN participants, but those differences were not statistically significant.

The prevalence of diabetes is increasing globally, and it causes $40 \%$ of CKD and $45 \%$ of ESRD. Moreover, ESRD 
A

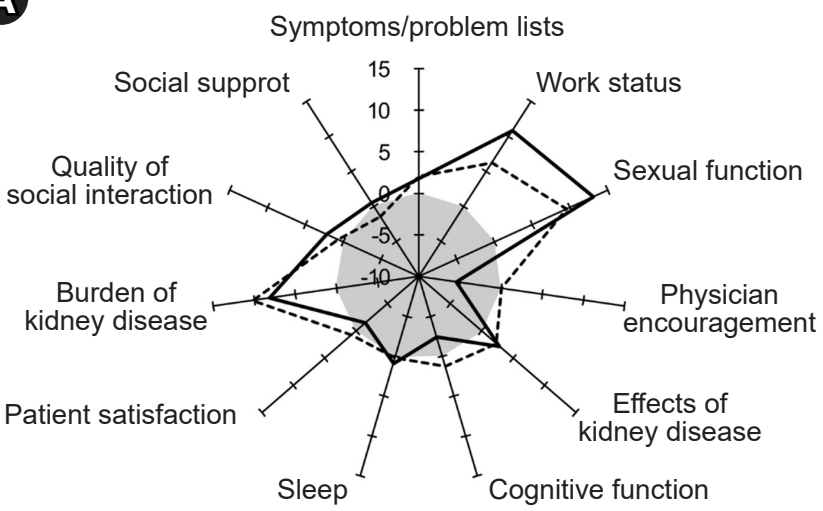

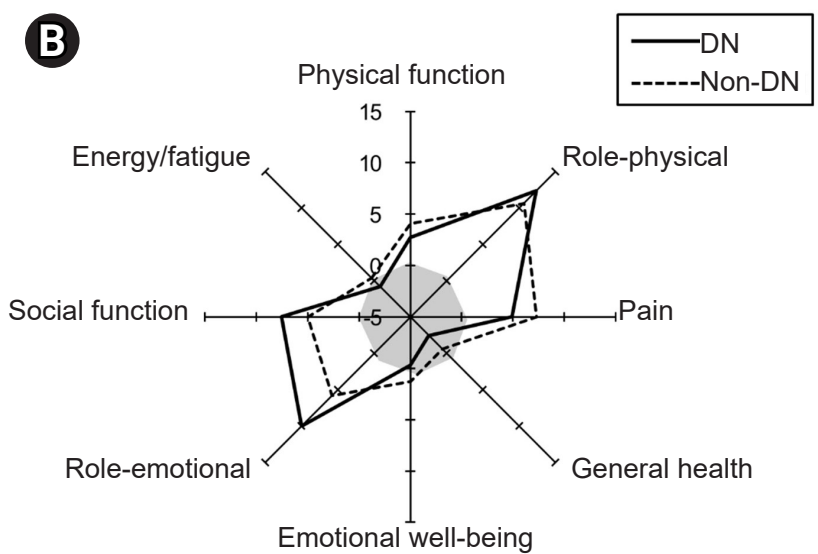

Emotional well-being

C

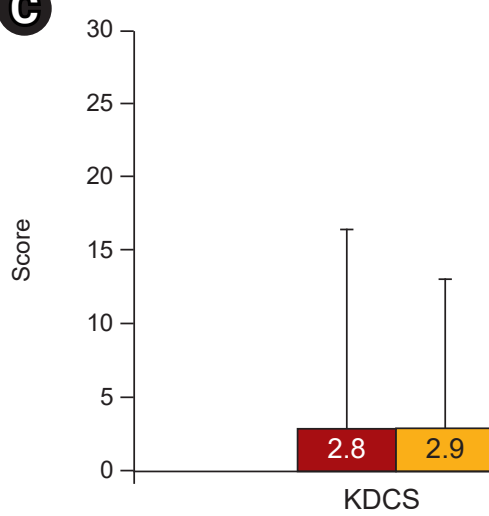

KDCS

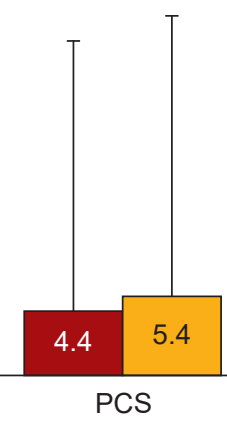

Figure 5. Spider chart of QoL changes. (A) Differences of each component of kidney disease component summary (KDCS) according to the two groups. (B) Differences of each component of mental component summary (MCS) and physical component summary (PCS) according to the two groups. (C) Summarizing KDCS, MCS, and PCS according to the two groups. Changes in QoL were analyzed in 221 participants who completed the 5-year follow-up QoL questionnaire. Relative to the gray circle marked 0, a positive value (outside) denotes decreased QoL, and a negative value (inside) indicates improved QoL. In both groups, several domain scores decreased over time, although the social support, patient satisfaction, and physician encouragement scores increased. The diabetic nephropathy (DN) patients had non-significantly greater decreases in the role-physical, social function, and role-emotional domains than non-DN patients. The composite scores decreased in both groups, with no statistically significant between-group difference.

QoL, quality of life.

caused by diabetes is $70 \%$ to $80 \%$ more costly than ESRD caused by other conditions. Therefore, the incidence of ESRD and its associated socioeconomic costs can be reduced by diagnosing and delaying the progression of $\mathrm{DN}$. The typical clinical progression of kidney disease in diabetes is microalbuminuria 3 years after diagnosis, overt albuminuria 15 years later, renal function decline another 5 years after that, and ESRD 5 years after renal function decline begins. DN can follow an atypical progression, but for the most part, preventing renal function from deteriorating to DN requires adequate control of diabetes, blood pressure, diet, and exercise before overt proteinuria occurs. Therefore, patients face considerable psychological and physical stress beginning with their diabetes diagnosis.

Patients are forced to follow a restricted diet, have to live with the burden of complying with medication instructions, and often experience the risk of hypoglycemia and hyperglycemia in their daily lives. As such, they experience difficulties that are unlike those experienced by patients with other types of CKD. Moreover, advanced DN is often caused by not adhering to those necessary guidelines and a long duration of diabetes, both of which can reduce QoL. In this study, QoL was generally lower in the DN group than in the nonDN group, and in some domains, that inferiority was statistically significant. Therefore, because of the prevalence and severity of disease, as well as patients' QoL, particular care 
should be taken to manage DN aggressively, and medical providers should be vigilant about the disease.

To summarize, in this study, DN patients had lower QoL scores than non-DN patients in the domains of the effects of kidney disease, cognitive function, and general health. The domain of the effects of kidney disease includes dietary restrictions due to kidney disease, the effects of kidney disease on working around the house or traveling, dependence on medical staff, stress and worries, and discomfort regarding one's sex life or personal appearance. Therefore, medical providers should establish realistic guidelines by providing detailed information about dietary restrictions. Moreover, social considerations are also needed to prevent diabetes from becoming a barrier to working around the house or traveling.

Providers should also help patients overcome the stress caused by the disease and establish an appropriate self-image. Proactive interventions to protect patients' sex lives also seem necessary. Regarding cognitive function, a rehabilitation program in collaboration with neurologists to prevent the loss of cognitive functions is needed. Lastly, in terms of general health, DN patients should be taught to have a positive perspective on their own health that allows them to cope with diabetes through proactive management, even though curing the condition is not a reasonable goal.

Chin et al. [9] found that in a cohort of Koreans older than 65 years, the glomerular filtration rate (using a cutoff of 45 $\mathrm{mL} / \mathrm{min} / 1.73 \mathrm{~m}^{2}$ ) influenced HRQoL. Moreover, they found that PCS scores were lower than MCS scores and that lower hemoglobin levels affected MCS (PCS, 54.5; MCS, 51.7). In another cohort, lower QoL was found to affect all-cause mortality [10]. Choi et al. [11] used the EQ-5D questionnaire in the Korea National Health and Nutrition Examination Survey and reported that low sodium excretion correlated with low QoL. Anemia, the number of teeth, sleeping time, vitamin D levels, socioeconomic status, arthritis, and depression were also found to correlate with QoL [12-16]. Kim et al. [17] found that QoL among elderly Korean peritoneal dialysis patients was better than that among elderly hemodialysis patients in some domains. The KNOW-CKD cohort study also found that lower vitamin D levels were associated with lower QoL scores [18] and that low PCS scores correlated with CKD progression [19].

Interestingly, in our study, higher platelet counts and alkaline phosphatase levels were associated with lower QoL.
According to Molnar et al. [20], a high platelet count is associated with renal cachexia and cardiovascular mortality. Our results can be interpreted similarly: hepcidin, a biomarker of inflammation, was significant in the univariate analysis, suggesting a connection between malnutrition-inflammation cachexia syndrome and low QoL. Alkaline phosphatase levels tend to increase as CKD progresses and are closely related to CKD mineral bone disease (MBD). A previous study reported that high alkaline phosphatase levels among ESRD patients were associated with hospitalization and death [21]. Therefore, it is possible that renal cachexia, inflammation, and CKD MBD, which are all reflected in the platelet count and alkaline phosphatase level, affected QoL.

A limitation of this study is that it was not possible to statistically control for all physical and social factors. However, we used all available resources to control for factors that affect QoL, though that required excluding many participants from the study. As described in the Methods section, PSM selected only around 500 patients from the original pool of more than 1,800 . The fact that more patients were not included in the analysis is a limitation. Moreover, the KDQOL-SF questionnaire was first developed for dialysis patients, so another limitation is that we could not use a questionnaire specifically designed for predialysis patients. However, the KDQOL-SF has been used in many other studies. This questionnaire was also completed through self-reporting by patients in the cohort, so the possibility of selection bias cannot be ruled out.

In our first analysis, we found significant QoL variables in DN. In the univariate analysis, cardiovascular disease was not a significant variable in QoL, and in the age-adjusted Charlson comorbidity index (CCI), a score of 8 points or higher was a significant factor in the PCS and KDCS scores. However, in the multivariate analysis, CCI lost its significance, and clopidogrel remained a significant variable. We suggest that that is why clopidogrel use itself is a stronger QoL variable than cardiovascular disease history or age-adjusted CCI.

In conclusion, we analyzed the factors that affect the QoL of DN patients using prospective cohort data and conducted additional comparative analyses of diabetes and QoL using PSM. A balance between the DN and non-DN groups was achieved through PSM. In the following analyses of QoL, few variables differed statistically between the two groups due to the issue of sample size, but most absolute scores were lower in the DN group. Some issues remain to be resolved 
before it can be conclusively stated that DN and non-DN patients do not differ significantly in QoL. Because the absolute QoL scores were lower among DN patients, we suggest that clinicians should be especially careful regarding QoL in this subgroup of patients.

\section{Conflicts of interest}

All authors have no conflicts of interest to declare.

\section{Funding}

This research was supported by the Research Program funded by the Korean Centers for Disease Control and Prevention (2011E3300300, 2012E3301100, 2013E3301600, 2013E3301601, 2013E3301602, 2016E3300200, and 2019E320100) and a grant from the National Research Foundation of Korea (grant number: NRF-2021R1I1A3057140).

\section{Acknowledgments}

The authors gratefully thank the clinical research staff and nurses of the KNOW-CKD study.

\section{Authors' contributions}

Conceptualization: HK, JL, KHO

Methodology: GHC, HK, KHO

Software: HMJ

Validation: SHK, JEG, JJY, JH

Formal analysis: $\mathrm{MH}$

Investigation: $\mathrm{HJK}, \mathrm{SHH}$

Resources: JL, YHK, JH

Data curation: SAS

Visualization: HK

Supervision, Project administration, Funding acquisition: KHO Writing-original draft: $\mathrm{HK}$, JWY

Writing-review \& editing: KHO

All authors read and approved the final manuscript.

\section{ORCID}

Hyunsuk Kim, https://orcid.org/0000-0003-1889-253X Joongyub Lee, https://orcid.org/0000-0003-2784-3772 Gwang Ho Choi, https://orcid.org/0000-0001-6443-5941
Hae Min Jeong, https://orcid.org/0000-0003-0497-6732 Seok hyung Kim, https://orcid.org/0000-0001-7605-082X Jae Eon Gu, https://orcid.org/0000-0003-2508-4418 Jeong-Ju Yoo, https://orcid.org/0000-0002-7802-0381 Miyeun Han, https://orcid.org/0000-0001-7304-2496 Hyo-Jin Kim, https://orcid.org/0000-0001-9289-9073 Su-Ah Sung, https://orcid.org/0000-0001-8434-9944 Seung Hyeok Han, https://orcid.org/0000-0001-7923-5635 Yeong Hoon Kim, https://orcid.org/0000-0002-4101-9993 Jong-Woo Yoon, https://orcid.org/0000-0002-7915-3733 Jongho Heo, https://orcid.org/0000-0002-6405-3860 Kook-Hwan Oh, https://orcid.org/0000-0001-9525-2179

\section{References}

1. Hays RD, Kallich JD, Mapes DL, et al. Kidney Disease Quality of Life Short Form (KDQOL-SF), version 1.3: a manual for use and scoring. Santa Monica, CA: Rand Corp.; 1997. p. 39.

2. Bradley C, Speight J. Patient perceptions of diabetes and diabetes therapy: assessing quality of life. Diabetes Metab Res Rev 2002;18 Suppl 3:S64-S69.

3. Cox D, Gonder-Frederick L, McCall A, Kovatchev B, Clarke W. The effects of glucose fluctuation on cognitive function and QOL: the functional costs of hypoglycaemia and hyperglycaemia among adults with type 1 or type 2 diabetes. Int J Clin Pract Suppl 2002;20-26.

4. Undén AL, Elofsson S, Andréasson A, Hillered E, Eriksson I, Brismar K. Gender differences in self-rated health, quality of life, quality of care, and metabolic control in patients with diabetes. Gend Med 2008;5:162-180.

5. Akinci F, Yildirim A, Gözü H, Sargin H, Orbay E, Sargin M. Assessment of health-related quality of life (HRQoL) of patients with type 2 diabetes in Turkey. Diabetes Res Clin Pract 2008;79:117-123.

6. Oh KH, Park SK, Park HC, et al. KNOW-CKD (KoreaN cohort study for Outcome in patients With Chronic Kidney Disease): design and methods. BMC Nephrol 2014;15:80.

7. Braitman LE, Rosenbaum PR. Rare outcomes, common treatments: analytic strategies using propensity scores. Ann Intern Med 2002;137:693-695.

8. D \'Agostino RB Jr. Propensity score methods for bias reduction in the comparison of a treatment to a non-randomized control group. Stat Med 1998;17:2265-2281.

9. Chin HJ, Song YR, Lee JJ, et al. Moderately decreased renal function negatively affects the health-related quality of life among 
the elderly Korean population: a population-based study. Nephrol Dial Transplant 2008;23:2810-2817.

10. Han SS, Kim KW, Na KY, et al. Quality of life and mortality from a nephrologist \'s view: a prospective observational study. BMC Nephrol 2009;10:39.

11. Choi HM, Lee KB, Kim H, Hyun YY. Sodium excretion and health-related quality of life: the results from the Korea National Health and Nutrition Examination Survey 2010-2011. Eur J Clin Nutr 2018;72:1490-1496.

12. Park HE, Song HY, Han K, Cho KH, Kim YH. Number of remaining teeth and health-related quality of life: the Korean National Health and Nutrition Examination Survey 2010-2012. Health Qual Life Outcomes 2019;17:5.

13. Kim YJ, Han KD, Cho KH, Kim YH, Park YG. Anemia and health-related quality of life in South Korea: data from the Korean national health and nutrition examination survey 2008-2016. BMC Public Health 2019;19:735.

14. Kim JH, Park EC, Yoo KB, Park S. The association between short or long sleep times and quality of life (QOL): results of the Korea National Health and Nutrition Examination Survey (KNHANES IV-V). J Clin Sleep Med 2015;11:625-634.

15. Kim HJ, Lee JY, Kim TJ, Lee JW. Association between serum vitamin D status and health-related quality of life (HRQOL) in an older Korean population with radiographic knee osteoarthritis: data from the Korean national health and nutrition examination survey (2010-2011). Health Qual Life Outcomes 2015;13:48.

16. Kwon KM, Lee JS, Jeon NE, Kim YH. Factors associated with health-related quality of life in Koreans aged over 50 years: the fourth and fifth Korea National Health and Nutrition Examination Survey. Health Qual Life Outcomes 2017;15:243.

17. Kim H, An JN, Kim DK, et al. Elderly peritoneal dialysis compared with elderly hemodialysis patients and younger peritoneal dialysis patients: competing risk analysis of a Korean prospective cohort study. PLoS One 2015;10:e131393.

18. Oh TR, Kim CS, Bae EH, et al. Association between vitamin D deficiency and health-related quality of life in patients with chronic kidney disease from the KNOW-CKD study. PLoS One 2017;12:e0174282.

19. Oh TR, Choi HS, Kim CS, et al. Association between health related quality of life and progression of chronic kidney disease. Sci Rep 2019;9:19595.

20. Molnar MZ, Streja E, Kovesdy CP, et al. High platelet count as a link between renal cachexia and cardiovascular mortality in end-stage renal disease patients. Am J Clin Nutr 2011;94:945954.

21. Blayney MJ, Pisoni RL, Bragg-Gresham JL, et al. High alkaline phosphatase levels in hemodialysis patients are associated with higher risk of hospitalization and death. Kidney Int 2008;74:655663. 